\title{
Sensitivity \& stability made easy? Sample introduction for the latest Neoma MC-ICP-MS.
}

\author{
GRANT CRAIG ${ }^{1}$, CLAUDIA BOUMAN ${ }^{2}$, NICHOLAS \\ LLOYD $^{2}$ AND JOHANNES SCHWIETERS ${ }^{1}$ \\ ${ }^{1}$ Thermo Fisher Scientific (Bremen) $\mathrm{GmbH}$ \\ ${ }^{2}$ Thermo Fisher Scientific \\ Presenting Author: grant.craig@thermofisher.com
}

In high precision isotope ratio measurements, counting statistics often results in the precision achieved being directly related to the number of counts detected. If the amount of sample available is finite then the only way to measure isotope ratios more precisely is to increase the sensitivity of the mass spectrometer. However many of the most popular methods to increase sensitivity can often come at a cost to stability.

To take multi-collector inductively coupled plasma mass spectrometry (MC-ICP-MS) technology further Thermo Fisher Scientific $^{\mathrm{TM}}$ have introduced the new Thermo Scientific ${ }^{\mathrm{TM}}$ Neoma ${ }^{\mathrm{TM}}$ MC-ICP-MS, blending cutting-edge and field proven technology from the Ultra ${ }^{\mathrm{TM}}$ HR-IRMS and iCAP Qnova ${ }^{\mathrm{TM}}$ Series ICP-MS ${ }^{1}$. When compared to the design of previous generation instruments, many of the enhancements in the Neoma MC-ICP-MS are to features, such as the ICP and vacuum system, which directly impact sensitivity and stability.

Here we report our investigations into using the Neoma MCICP-MS with the wide variety of sample introduction used in MC-ICP-MS, such as desolvating nebulizer systems and laser ablation. The greater level of integration of sample introduction systems with the Neoma MC-ICP-MS, using the Qtegra ${ }^{\text {TM }}$ ISDS software, made it significantly easier to achieve optimum conditions. Sensitivity and high precision isotope ratio measurements were made for elements across the periodic table. By using a comprehensive approach, comparisons between the different sample introduction systems, and between Neoma MCICP-MS and previous generation MC-ICP-MS were constructed. These demonstrated that with the Neoma MC-ICP-MS, high precision isotope ratio data is within the reach of every user.

[1] Thermo Fisher Scientific, 2020, BR30600-EN 0520C: Neoma Multicollector ICP-MS [pdf], Thermo Fisher Scientific. 